Original Article

\title{
The Comparison of Long-Term Results between Aortofemoral and Axillofemoral Bypass for Patients with Aortoiliac Occlusive Disease
}

\author{
Kimihiro Igari, MD, PhD, Toshifumi Kudo, MD, PhD, Sotaro Katsui, MD, PhD, \\ Masato Nishizawa, MD, PhD, and Hiroyuki Uetake, MD, PhD
}

\begin{abstract}
Purpose: We evaluated the clinical outcomes of aortofemoral bypass (AoFB) and axillofemoral bypass (AxFB) surgeries for complex aortoiliac occlusive disease (AIOD) and compared them from the perspectives of safety and efficacy. Methods: We retrospectively reviewed 21 patients with AoFB grafting and 9 patients with AxFB grafting. The demographic information of the patients was examined, and the intra-, peri-, and postoperative results as well as long-term outcomes were evaluated. Results: In the AoFB, 2 of $21(9.5 \%)$ cases had intra- and perioperative complications, and 4 of $21(19.0 \%)$ cases had postoperative complications; however, there were no postoperative mortalities. In the AxFB, two of nine (22.2\%) cases had postoperative graft thrombosis; however, again there were no postoperative mortalities. According to KaplanMeier analysis, the primary patency rates in the AoFB and AxFB groups at 5 years were $\mathbf{9 4 . 8 \%}$ and $53.6 \%$, respectively $(P=0.001)$, while the limb salvage rates at 5 years were 96.4\% and $92.9 \%$, respectively $(P=0.320)$.

Conclusions: Even though the patency rates with AxFB grafting were inferior to those with AoFB grafting, AxFB was able to achieve equivalent limb salvage rates and should thus be considered as an alternative treatment method, especially when limb salvage is a goal.
\end{abstract}

Keywords: aortoiliac occlusive disease, aortofemoral bypass, axillofemoral bypass

\section{Introduction}

Aortoiliac occlusive disease (AIOD) is a challenging disease that should be treated by aortofemoral bypass

Department of Specialized Surgery, Tokyo Medical and Dental University, Tokyo, Japan

Received: November 11, 2019; Accepted: February 15, 2020 Corresponding author: Kimihiro Igari, MD, PhD. Department of Specialized Surgery, Tokyo Medical and Dental University, 1-5-45 Yushima, Bunkyo-ku, Tokyo 113-8519, Japan

Email: igari.srg1@tmd.ac.jp

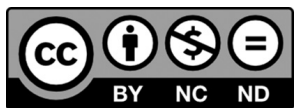

This work is licensed under a Creative Commons Attribution-NonCommercialNoDerivatives International License.

(C)2020 The Editorial Committee of Annals of Thoracic and Cardiovascular Surgery
(AoFB); however, the relatively high rates of peri- and postoperative morbidities and mortalities, especially for older and/or high-risk patients, with AoFB remain a concern. ${ }^{1)}$ According to the 2017 ESC guidelines, ${ }^{2)}$ in patients with complex AIOD (TASC II ${ }^{3)}$ D lesions) who are fit for surgery, AoFB should be considered, and extra-anatomical bypass, including axillofemoral bypass $(\mathrm{AxFB})$, may be indicated for patients with no other alternatives for revascularization. Traditionally, AxFB has been associated with a lower primary patency rate (67.7\% at 5 years) than AoFB (88.5\% at 5 years). ${ }^{4)}$ However, while AxFB has been applied for challenging cases with severe comorbidities, including for older patients than typically receive AoFB, the patients treated by AxFB have shown a lower overall survival rate $(67.0 \%$ at 1 year) than those who undergo AoFB. ${ }^{5)}$ Therefore, it 
might be difficult for AxFB to be considered as a viable alternative treatment to AoFB.

The present study includes a retrospective review of our clinical experiences with bypass surgeries for patients with complex AIOD (defined as TASC II D lesions) and evaluates the long-term outcomes, including patency rates. We also hypothesized that AxFB might be an alternative treatment way to AoFB, with comparable good postoperative outcomes and lower peri- and postoperative morbidities and mortalities.

\section{Materials and Methods}

This study was a retrospective study of the data obtained from consecutive patients treated between April 2008 and September 2018. All surveys and consent forms were approved by the Institutional Review Board (IRB No. M2019-044) of Tokyo Medical and Dental University Hospital. During this period, 21 AoFB procedures were performed in 21 patients, and $9 \mathrm{AxFB}$ procedures were performed in 9 patients. During the same period, we performed 10 endovascular treatment for patients with complex AIOD; however, we did not include these 10 cases from this study.

\section{Patients}

All patients in this study were diagnosed with complex AIOD (defined as TASC II D lesions) by computed tomography angiography (CTA), duplex ultrasonography (DUS) and/or magnetic resonance angiography (MRA). This study excluded patients with AIOD due to Buerger disease, acute aortic occlusion, and other kinds of systemic conditions except for atherosclerotic disease. The data from all interventions were retrospectively collected in a dedicated database that included the demographic data, preoperative state of the patients, results of the clinical and diagnostic assessments, intraoperative findings, and follow-up outcomes. ${ }^{6}$ Clinical evaluations assessed the ankle brachial pressure index (ABI), and the Rutherford classification was used for chronic ischemia. ${ }^{6}$ We preoperatively evaluated the outflow vessels, which were classified by TASC II femoropopliteal lesions. ${ }^{3)}$

\section{Surgical techniques}

Open surgical repair was indicated for patients with symptoms more severe than Rutherford category 2 in unilateral or bilateral limbs. The choice for surgical procedure (AoFB or AxFB) for each patient was not randomized, but depended on the decision by each operating surgeon. However, choice of surgical procedure depended on the patient conditions, including comorbidities. In brief, AxFB was preferred for patients with relatively contraindicated operative aspects, such as a history of abdominal surgery. As there were no specific criteria for selecting surgical techniques, we discussed the surgical methods for each case preoperatively.

All AoFB procedures were performed through the transperitoneal approach under general anesthesia. All cases were bilateral with Dacron grafts. Proximal anastomoses were typically performed at the infrarenal aorta in an end-to-side manner with or without suprarenal cross clamping. For cases with severe calcification at the proximal anastomosis site, endarterectomy was added. The distal anastomosis was constructed to the common/ external iliac or common/deep femoral arteries according to the pattern of occlusive disease at the groin. All distal anastomoses were performed in an end-to-side fashion with or without endarterectomy.

All AxFB procedures except for one case were performed under general anesthesia. The remaining case was performed under local anesthesia with sedation. All AxFB procedures were bilateral using an externally supported 10-mm-diameter main limb of a Dacron graft with 8-mm-diameter externally supported bilateral femoral components of Dacron grafts. These bypass grafts were positioned subcutaneously, and proximal anastomoses were usually made at the right side of the axillary artery in an end-to-side manner. In the same manner as for AoFB, the distal anastomosis of AxFB was constructed to the common/deep femoral arteries, depending on the pattern of the atherosclerotic lesions at the groin, in an end-to-side fashion with or without endarterectomy.

\section{Postoperative management and the follow-up protocol}

After the surgery, most patients received antiplatelet agents, except for the cases contraindicated for these kinds of medications. Postoperative complications were those that occurred within 30 days after the operation and were analyzed in terms of the mortality, morbidity, and graft thrombosis. The postoperative surveillance included clinical examinations, consisting of ABI measurements and clinical symptoms according to the Rutherford category. Patients were checked every 3 months for the first year and subsequently every 3-6 months during the follow-up period.

Graft patency was assessed by the palpation of the femoral arterial pulse. When graft stenosis or occlusion 
was suspected, CTA, DUS, and/or MRA were performed to examine the patency of the implanted graft. Primary patency was defined as an uninterrupted flow with neither intervention required to treat disease progression in the adjacent native vessel nor any additional procedure performed. Assisted primary patency was defined as patency of the bypass graft with additional procedures required to maintain the patency of the graft before graft occlusion. Secondary patency was defined as restored patency through the original treated segment with additional treatment after graft occlusion. Limb salvage was defined as the avoidance of major amputation above or below the knee. These patency and salvage rates were analyzed by the Kaplan-Meier method.

\section{Statistical analysis}

Statistical analyses were performed using the SPSS software program, version 22 (SPSS Inc., Chicago, IL, USA). Continuous variables were expressed as the median and interquartile range (IQR) and compared with the Mann-Whitney $\mathrm{U}$ test. The categorical variables were expressed as frequencies and percentages and assessed using the chi-squared test. $\mathrm{P}$ values of $<0.05$ were considered to indicate statistical significance. The rates of patency and limb salvage were analyzed using the Kaplan-Meier life-table analysis with log-rank tests to compare the groups.

\section{Results}

\section{Patient demographics}

The patients who underwent AoFB were more frequently complicated with dyslipidemia (14 patients, $66.7 \%, \mathrm{P}=0.025)$ than were those who underwent $\mathrm{AxFB}$ (2 patients, $22.2 \%$ ). In contrast, the patients who underwent $A x F B$ showed significantly lower values for the BMI $\left(19.5 \mathrm{~kg} / \mathrm{m}^{2}, \mathrm{P}=0.002\right)$ and hemoglobin $(12.4 \mathrm{~g} / \mathrm{dL}$, $\mathrm{P}=0.024)$ than those who underwent AoFB $\left(23.3 \mathrm{~kg} / \mathrm{m}^{2}\right.$ and $13.9 \mathrm{~g} / \mathrm{dL}$, respectively). There were no significant differences in patients' background characteristics between the groups. Furthermore, we calculated preoperative glomerular filtration rate (GFR), and defined the severity of chronic kidney disease (CKD) according to the value of GFR; G1 $>90 \mathrm{~mL} / \mathrm{min} / 1.73 \mathrm{~m}^{2}$, G2 $60-89 \mathrm{~mL} / \mathrm{min} / 1.73 \mathrm{~m}^{2}$, G3a $45-59 \mathrm{~mL} / \mathrm{min} / 1.73 \mathrm{~m}^{2}$, G3b $30-44 \mathrm{~mL} / \mathrm{min} / 1.73 \mathrm{~m}^{2}$, G4 $15-29 \mathrm{~mL} / \mathrm{min} / 1.73 \mathrm{~m}^{2}$, and $\mathrm{G} 5<15 \mathrm{~mL} / \mathrm{min} / 1.73 \mathrm{~m}^{2}{ }^{2}$ ) In the AoFB group, there were 15 patients (71.4\%) with CKD G1 or 2, and 6 patients (28.6\%) with CKD G3a or $\mathrm{G} 3 \mathrm{~b}$. In the AxFB group, there were five patients (55.6\%) with CKD G1 or 2, and four patients (44.4\%) with CKD G3a or G3b or G4. There was no significant difference of the severity of CKD between AoFB and AxFB $(\mathrm{P}=0.398)$.

We evaluated the patients' preoperative physical status according to the American Society of Anesthesiologists (ASA) - Physical Status Classification system. ${ }^{8)}$ We assumed the preoperative levels of activity of daily living (ADL) by classifying walking status as below; walking without any assistance, walking or moving with some assistance (including wheelchair), and bedridden. In the AoFB group, there were 14 patients (66.7\%) with ASA class II, and 7 patients (33.3\%) with ASA class III. In the AxFB group, there were three patients (33.3\%) with ASA class II, and six patients (66.7\%) with ASA class III. Although the patients in AxFB group showed the trend of preoperative poor physical condition, it showed no significant difference $(\mathrm{P}=0.091)$. In the perspective of preoperative ADL level, 19 patients (90.5\%) could walk without any assistance in the AoFB group; however, only 4 patients (44.4\%) could walk without any assistance in the AxFB group, which showed statistically significant difference $(\mathrm{P}=0.006)$. Regarding patients' preoperative clinical status, the patients treated by AoFB showed higher $\mathrm{ABI}$ values $(0.52, \mathrm{P}<0.001)$ and lower categories of Rutherford clinical classification than those treated by AxFB (0.31). This indicates that the patients who underwent AxFB had a more severe ischemic condition in the lower limbs than those who underwent AoFB. There was no statistically significant difference in the rate of TASC II femoropopliteal lesions between the groups (Table 1).

\section{Surgical details}

The patients who underwent AoFB had a significantly longer operative time $(295 \mathrm{~min}, \mathrm{P}<0.001)$ and more intraoperative blood loss $(1171 \mathrm{~mL}, \mathrm{P}<0.001)$ than those who underwent AxFB (195 min and $182 \mathrm{~mL}$, respectively). In the AoFB group, all of the proximal anastomosis sites were at the infrarenal level of the aorta, and 6 of 21 patients (28.6\%) needed suprarenal cross-clamping to create a proximal anastomosis. The distal anastomosis sites were mainly located at the common femoral arteries (20 limbs of 42 limbs, 47.6\%) and external iliac arteries (18 limbs of 42 limbs, 42.8\%). Two limbs of 42 limbs $(4.8 \%)$ were anastomosed to common iliac arteries, and 2 limbs of 42 limbs (4.8\%) were done with deep femoral arteries. In the AxFB group, seven of nine proximal anastomosis sites were at the right side of the 
Table 1 Patient demographics

\begin{tabular}{|c|c|c|c|}
\hline Variables & $\operatorname{AoFB}(n=21)$ & $\operatorname{AxFB}(n=9)$ & $P$ value \\
\hline Age (years) & $70(64-74)$ & $78(66-84)$ & 0.112 \\
\hline Gender (male) & $19(90.4 \%)$ & $7(77.8 \%)$ & 0.348 \\
\hline BMI $\left(\mathrm{kg} / \mathrm{m}^{2}\right)$ & $23.3(21.7-25.1)$ & $19.5(18.5-20.0)$ & 0.002 \\
\hline \multicolumn{4}{|l|}{ Comorbidities } \\
\hline Hypertension & $16(76.2 \%)$ & $8(88.9 \%)$ & 0.426 \\
\hline Diabetes mellitus & $11(52.4 \%)$ & $2(22.2 \%)$ & 0.127 \\
\hline Dyslipidemia & $14(66.7 \%)$ & $2(22.2 \%)$ & 0.025 \\
\hline Coronary artery disease & $4(19.0 \%)$ & $2(22.2 \%)$ & 0.842 \\
\hline Cerebrovascular disease & $4(19.0 \%)$ & $4(44.4 \%)$ & 0.397 \\
\hline Chronic obstructive pulmonary disease & $2(9.5 \%)$ & $1(11.1 \%)$ & 0.894 \\
\hline Smoking habit & $19(90.4 \%)$ & $6(66.7 \%)$ & 0.109 \\
\hline \multicolumn{4}{|l|}{ Laboratory test } \\
\hline Hemoglobin $(\mathrm{g} / \mathrm{dL})$ & $13.9(12.9-15.1)$ & $12.4(10.6-13.1)$ & 0.024 \\
\hline Albumin $(\mathrm{g} / \mathrm{dL})$ & $4.1(3.9-4.3)$ & $4.0(3.5-4.1)$ & 0.159 \\
\hline Creatinine $(\mathrm{mg} / \mathrm{dL})$ & $0.90(0.75-1.11)$ & $0.82(0.70-0.92)$ & 0.587 \\
\hline GFR $\left(\mathrm{mL} / \mathrm{min} / 1.73 \mathrm{~m}^{2}\right)$ & $64.18(50.72-83.82)$ & $65.95(46.15-84.78)$ & 0.952 \\
\hline Severity of CKD & & & 0.398 \\
\hline $\mathrm{G} 1,2$ & $15(71.4 \%)$ & $5(55.6 \%)$ & \\
\hline G3a, 3b, 4 & $6(28.6 \%)$ & $4(44.4 \%)$ & \\
\hline \multicolumn{4}{|l|}{ Preoperative physical status } \\
\hline ASA Physical Status Classification & & & 0.0091 \\
\hline Class II & $14(66.7 \%)$ & $3(33.3 \%)$ & \\
\hline Class III & $7(33.3 \%)$ & $6(66.7 \%)$ & \\
\hline ADL level & & & 0.006 \\
\hline Walking without any assistance & $19(90.5 \%)$ & $4(44.4 \%)$ & \\
\hline Walking or moving with some assistance & $2(9.5 \%)$ & $5(55.6 \%)$ & \\
\hline Preoperative clinical status & $\mathrm{n}=42$ & $\mathrm{n}=18$ & \\
\hline Ankle brachial pressure index & $0.52(0.42-0.64)$ & $0.31(0.20-0.39)$ & $<0.001$ \\
\hline Rutherford classification & & & $<0.001$ \\
\hline 0 & $1(2.4 \%)$ & $0(0 \%)$ & \\
\hline $1-3$ & $40(95.2 \%)$ & $7(38.9 \%)$ & \\
\hline 4 & $0(0 \%)$ & $5(27.8 \%)$ & \\
\hline $5-6$ & $1(2.4 \%)$ & $6(33.3 \%)$ & \\
\hline Femoropopliteal lesion & & & 0.317 \\
\hline TASC II 0 & $29(69.1 \%)$ & $10(55.5 \%)$ & \\
\hline TASC II A, B & $8(19.0 \%)$ & $3(16.7 \%)$ & \\
\hline TASC II C, D & $5(11.9 \%)$ & $5(27.8 \%)$ & \\
\hline
\end{tabular}

ADL: activity of daily living; AoFB: aortofemoral bypass; ASA: American Society of Anesthesiologists;

AxFB: axillofemoral bypass; BMI: body mass index; CKD: chronic kidney disease; GFR: glomerular filtration rate; TASC II: Trans-Atlantic Inter-society Consensus document

axillary artery, while the other two were at the left side. The most common distal anastomosis site was the common femoral arteries (14 limbs of 18 limbs, 77.8\%), and all other sites were located at the deep femoral arteries (4 limbs of 18 limbs, 22.2\%), where we performed additional profundoplasty (Table 2).

\section{Postoperative outcomes}

In the AoFB group, there were six patients with postoperative complications (28.6\%), including one case $(4.8 \%)$ of intraoperative thrombosis in implanted graft limb, and one case $(4.8 \%)$ of postoperative bleeding.
These two patients were needed additional procedures, thrombectomy and hemostasis, for improvement. The other four patients with postoperative complication were improved with conservative treatment. In the AxFB group, there were two patients with postoperative complications, both with thrombosis of the implanted graft (one unilateral and one bilateral). The unilateral thrombosed graft was treated by thrombectomy; however, the treated graft became occluded again, and no further treatment was applied. The other case had a severe systemic condition, and the patient did not desire any further treatment, including thrombectomy, so the bilateral 
Table 2 Operative details and postoperative outcomes

\begin{tabular}{lccr}
\hline Variables & AoFB $(\mathrm{n}=21)$ & AxFB $(\mathrm{n}=9)$ & P value \\
\hline Operative time (min) & $295(278-333)$ & $195(174-233)$ & $<0.001$ \\
Intraoperative blood loss (mL) & $171(926-1462)$ & $182(147-248)$ & $<0.001$ \\
Intraoperative blood transfusion & $5(23.8 \%)$ & $1(11.1 \%)$ & 0.426 \\
Postoperative complications & $6(28.6 \%)$ & $2(22.2 \%)$ & 0.719 \\
$\quad$ Intraoperative acute thrombosis & $1(4.8 \%)$ & & \\
Postoperative bleeding & $1(4.8 \%)$ & & \\
Acute kidney injury & $1(4.8 \%)$ & & \\
Wound infection & $1(4.8 \%)$ & & \\
Ileus & $1(4.8 \%)$ & & \\
Pneumonia & $1(4.8 \%)$ & $2(22.2 \%)$ & \\
Postoperative thrombosis & $\mathrm{n}=42$ & $\mathrm{n}=18$ & 0.724 \\
Postoperative clinical status & $1.04(0.95-1.12)$ & $0.78(0.68-0.88)$ & \\
ABI value & $0.49(0.38-0.63)$ & $0.46(0.37-0.61)$ & \\
Increase value of ABI & & & \\
Rutherford classification & $29(69.0 \%)$ & $4(22.2 \%)$ & \\
$\quad 0$ & $13(31.0 \%)$ & $11(61.1 \%)$ & \\
$1-3$ & $0(0 \%)$ & $0(0 \%)$ & \\
4 & $0(0 \%)$ & $3(16.7 \%)$ & \\
$\quad 5-6$ & $3(2-3)$ & $1(1-2)$ & 0.018 \\
Improvement level of Rutherford grade &
\end{tabular}

ABI: ankle brachial pressure index; AoFB: aortofemoral bypass; AxFB: axillofemoral bypass

grafts remained occluded. During the follow-up period, we evaluated the patients' clinical condition. The postoperative $\mathrm{ABI}$ was significantly higher in the AoFB group (1.04, $\mathrm{P}<0.001)$ than in the AxFB group (0.78); however, the postoperative increase in the ABI did not differ significantly between the AoFB and AxFB groups (0.49 and 0.46 , respectively; $P=0.724)$. Furthermore, the postoperative clinical status evaluated by the Rutherford classification was significantly better in the AoFB group (Grade 0; 29 limbs, 69.0\%) than in the AxFB group (Grade 1-3; 11 limbs, 61.1\%), and the improvement in the Rutherford clinical classification was significantly greater in the AoFB group $(3, \mathrm{P}=0.018)$ than in the AxFB group (1) (Table 2).

\section{Follow-up outcomes}

During the follow-up period (AoFB group; median 54 months, IQR 31-74 months, AxFB group; median 7 months, IQR 3-80 months), we evaluated the survival, patency, and limb salvage rates. There were three deaths $(14.3 \%)$ in the AoFB group; one was due to pneumonia, following sepsis, one was due to perioperative death for thoracic aortic aneurysm, and the other was due to unknown origin. On the other hand, there was no death in the AxFB group. In the AoFB group, 40 of $42 \mathrm{limbs}$ $(95.2 \%)$ retained their primary patency, and the remaining two showed assisted primary and secondary patency (1 each; $2.4 \%$ and 2.4\%). In the AxFB group,
13 of 18 limbs (72.2\%) retained their primary patency, and 2 others (11.1\%) showed secondary patency. The remaining three limbs became occluded. Regarding the limb salvage, 1 of 42 limbs (2.4\%) in the AoFB group had to be amputated due to further lower ischemia caused by infrainguinal lesions; however, both bypass grafts were patent. In the AxFB group, 1 of 18 limbs (5.6\%) had to be amputated because of gangrene that had not been improved by AxFB surgery.

According to the Kaplan-Meier analysis, the primary patency rates in the AoFB and AxFB groups were $97.6 \%$ and $83.3 \%$ at 1 year, $94.8 \%$ and $71.4 \%$ at 2 years, and $94.8 \%$ and $53.6 \%$ at 5 years, respectively (Log Rank $\mathrm{P}=0.001)$ (Fig. 1). The assisted primary patency rates in the AoFB and AxFB groups were $97.6 \%$ and $83.3 \%$ at 1 year, $97.6 \%$ and $71.4 \%$ at 2 years, and $97.6 \%$ and $53.6 \%$ at 5 years, respectively ( $\log \operatorname{Rank} \mathrm{P}<0.001)$. The secondary patency rates in the AoFB and AxFB groups were $100 \%$ and $83.3 \%$ at 5 years, respectively (Log Rank $\mathrm{P}=0.007)$. The limb salvage rates in the AoFB and AxFB groups were $96.4 \%$ and $92.9 \%$ at 5 years, respectively (Log Rank $\mathrm{P}=0.320)$ (Fig. 2).

\section{Discussion}

In the present study, we compared the surgical results of $\mathrm{AoFB}$, including the postoperative complications, to those of AxFB. AoFB is more frequently associated with 


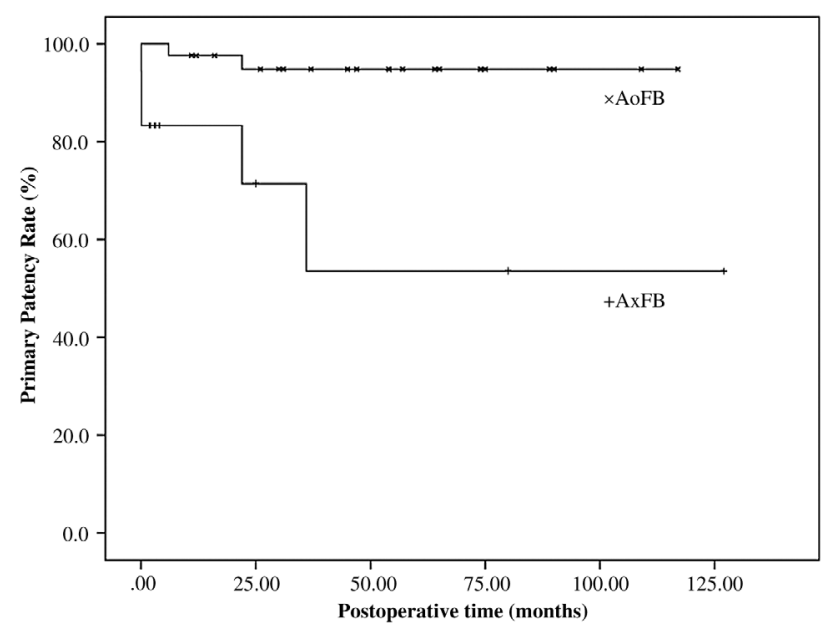

Fig. 1 Kaplan-Meier estimate of primary patency rate after 21 aortofemoral bypass (AoFB) and 9 axillofemoral bypass (AxFB) surgeries. The 5-year primary patency rate was significantly higher in AoFB than AxFB $(94.8 \%$ versus $53.6 \%$, Log Rank $\mathrm{P}=0.001$ ).

peri- and postprocedural complications than AxFB. ${ }^{9)}$ Therefore, we preferred AxFB to AoFB for patients with severe comorbidities. In the AxFB group, even though we had two cases $(22.2 \%)$ of early thrombosed bypass graft, there were no severe postoperative complications, including no mortalities. This might have been related to our surgical strategy, as we avoided performing AoFB in patients with challenging comorbidities. In contrast, in the AoFB group, there were six cases (28.6\%) with postoperative complications. However, all cases were able to be discharged without mortality. We demonstrated not only the efficacy but also the safety of AoFB in this study. Whether AoFB or AxFB is more appropriate should be considered on a case-by-case basis.

During the follow-up period, there were three deaths in the AoFB group; however, there was no death in the AxFB group. Generally, the patients with AxFB grafting showed lower survival rate compared to the patients with AoFB grafting. As same as our study population, the patients with AxFB showed poorer general physical condition with comorbidities preoperatively, which could be related to poorer survival rate. ${ }^{10)}$ In our AxFB group, the median follow-up duration was not so long (only 7 months, IQR 3-80 months); therefore, the shorter follow-up might be related to the low occurrence of death during the follow-up period.

It has been widely known that the long-term patency rate of AoFB is superior to that of AxFB. ${ }^{4,11)}$ The patency rates, including the rates of primary, assisted primary, and secondary patency, were inferior in the AxFB group to

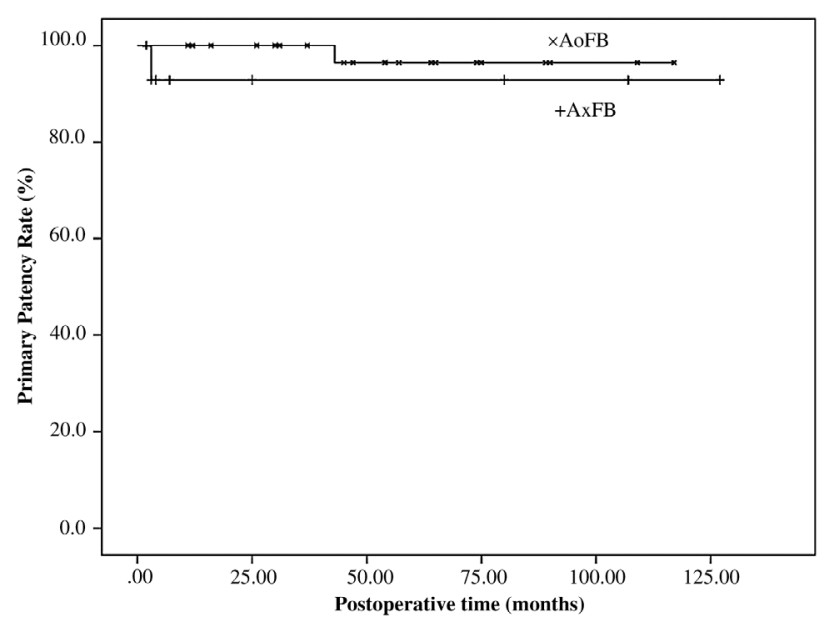

Fig. 2 Kaplan-Meier estimate of limb salvage rate after 21 aortofemoral bypass (AoFB) and 9 axillofemoral bypass (AxFB) surgeries. The 5-year limb salvage rate was similar between AoFB group and AxFB group (96.4\% and 92.9\%, Log Rank P = 0.320).

those in the AoFB group in the present study. However, we noted no significant difference in the limb salvage rate between the AxFB group (92.9\% at 5 years) and the AoFB group (96.4\% at 5 years). Passman et al. ${ }^{12}$ ) reported that the 5-year limb salvage rate with AxFB grafting did not differ markedly from that with AoFB grafting (89.0\% vs. 79.0\%). Furthermore, they found more severe limb ischemic conditions in the AxFB group (79.6\% with Rutherford classification 4-6) than in the AoFB group (41.7\% with Rutherford category 4-6). Similar to that report, we noted no significant inferiority in the limb salvage rate in the AxFB group, which had more severe preoperative ischemia in the lower limbs, compared with the AoFB group. This means that AxFB is the more favorable treatment option for achieving limb salvage, especially for patients with severe comorbidities and poor limb perfusion.

Several limitations associated with the present study warrant mention. Our study involved a small sample size, which might have affected the statistical significance. Furthermore, with a larger sample, we would have been able to analyze the beneficial factors for graft patency and limb savage using a logistic regression analysis. Unfortunately, we were unable to identify any statistically beneficial factors for good outcomes, including long-term patency. Furthermore, we evaluated only nine cases of AxFB grafting. We need to examine more cases with AxFB grafting, which might lead to a more meaningful discussion and consideration. We should collect more cases to confirm the safety of AoFB and the efficacy of AxFB in future cohort studies. 


\section{Conclusion}

We demonstrated that, for complex AIOD cases, AoFB grafting could be performed safely without severe postoperative complications, including mortality. The patency rates, including primary, assisted primary and secondary patency, in the AoFB group were superior to those in the AxFB group; however, the limb salvage rate in the AxFB group was not inferior to that in the AoFB group. Therefore, among patients with AIOD, AxFB should be considered as an alternative treatment to AoFB for achieving limb salvage, especially for patients with severe systemic comorbidities. We should conduct further studies to identify the beneficial factors of AoFB and AxFB grafting for complex AIOD cases.

\section{Disclosure Statement}

The authors have no conflicts of interest to declare.

\section{References}

1) McPhee JT, Madenci A, Raffetto J, et al. Contemporary comparison of aortofemoral bypass to alternative inflow procedures in the Veteran population. J Vasc Surg 2016; 64: 1660-6.

2) Aboyans V, Ricco JB, Bartelink MEL, et al. 2017 ESC guidelines on the diagnosis and treatment of peripheral arterial diseases, in collaboration with the European Society for Vascular Surgery (ESVS). Eur J Vasc Endovasc Surg 2018; 55: 305-68.
3) Norgren L, Hiatt WR, Dormandy JA, et al. Inter-society consensus for the management of peripheral arterial disease (TASC II). Eur J Vasc Endovasc Surg 2007; 33: $\mathrm{S} 1-75$.

4) Onohara T, Komori K, Kume M, et al. Multivariate analysis of long-term results after an axillobifemoral and aortobifemoral bypass in patients with aortoiliac occlusive disease. J Cardiovasc Surg (Torino) 2000; 41: 905-10.

5) Nguyen KP, Perrone KH, Rahman A, et al. The role of axillofemoral bypass in current vascular surgery practice. Am J Surg 2016; 211: 968-71.

6) Rutherford RB, Baker JD, Ernst C, et al. Recommended standards for reports dealing with lower extremity ischemia: revised version. J Vasc Surg 1997; 26: 517-38.

7) Webster AC, Nagler EV, Morton RL, et al. Chronic kidney disease. Lancet 2017; 389: 1238-52.

8) Hurwitz EE, Simon M, Vinta SR, et al. Adding examples to the ASA-physical status classification improves correct assignment to patients. Anesthesiology 2017; 126: 614-22.

9) Clair DG, Beach JM. Strategies for managing aortoiliac occlusions: access, treatment and outcomes. Expert Rev Cardiovasc Ther 2015; 13: 551-63.

10) Samson RH, Showalter DP, Lepore MR, et al. Improved patency after axillofemoral bypass for aortoiliac occlusive disease. J Vasc Surg 2018; 68: 1430-7.

11) Hertzer NR, Bena JF, Karafa MT. A personal experience with direct reconstruction and extra-anatomic bypass for aortoiliofemoral occlusive disease. J Vasc Surg 2007; 45: 527-35, discussion 535.

12) Passman MA, Taylor LM, Moneta GL, et al. Comparison of axillofemoral and aortofemoral bypass for aortoiliac occlusive disease. J Vasc Surg 1996; 23: 263-9; discussion 269-71. 